\title{
Educación cívica en arquitectura y urbanismo: ciudadanos conscientes y participativos en la conformación de nuestro medio ambiente construido.
}

Camila Barreau Daly

Juan Luis Torres Galvez

Indice

Introducción

1.- Objetivos e hipótesis

2.- Aspectos pedagógicos

3.- Experiencia

4.- Resultados

5.- Reflexiones finales

6.- Bibliografía

\section{Resumen}

Educar en arquitectura en la niñez, durante el proceso de comprensión del Medio Ambiente Construido (MAC), permitiría constituir un proceso educativo para conformar ciudadanos más conocedores e interesados por el MAC, que manejen conceptos y habilidades que les permitan ser partícipes de su construcción. La existencia de ciudadanos con conciencia en arquitectura, fortalecería los lazos entre los actores participantes de la creación del MAC, permitiendo un mejor diálogo en las instancias de toma de decisiones que le dan forma.

Palabras claves: INTRODUCCIÓN DE LA ARQUITECTURA EN LA FORMACIÓN INFANTIL. EDUCACIÓN CÍVICA EN ARQUITECTURA. PIHLA MESKANEN. ANNU PUURUNEN. ILPO VUORELA.

\begin{abstract}
Teaching architecture to children, still in the process of understanding their Built Environment, would enable an educative process leading to greater knowledgment and interest in this topic among citizens, and therefore allow them to participate in its development. The existance of citizens with a developed consciousness of architecture would strengthen the relation between those participating in the creation of the Built Environment, thus allowing a better dialogue in the decisionmaking instances involved in this process.
\end{abstract}

Key words: TEACHING OF ARCHITECTURE AND URBANISM TO CHILDREN. PIHLA MESKANEN. ANNU PUURUNEN. ILPO VUORELA.

\section{Introducción}

Tras realizar una búsqueda sobre los sistemas de participación ciudadana en los procesos de planificación urbana en Finlandia, nos llamó especialmente la atención un planteamiento educativo creado por arquitectos en 1993, cuya forma de enfocar el problema de la participación ciudadana era muy diferente a la que conocemos en nuestro país. El planteamiento, si bien no era nuevo, centraba la atención en un área no pensada por nosotros, la educación cívica en arquitectura orientada a niños en edad escolar. La claridad con que se planteaba este tipo de educación nos motivó a indagar sobre este tema, a ponernos en contacto con quienes comenzaron este modelo y a conocer algunos de los centros de educación en arquitectura, así como las diferencias de enfoque entre ellos. 
El planteamiento finés consistía en educar desde la niñez a la ciudadanía sobre temas de arquitectura, con el fin de formar mejores ciudadanos, conscientes de la importancia de su MAC y del rol que recae sobre cada uno de ellos respecto de su cuidado y mejoramiento.

El interés de llevar en nuestro contexto los temas de la arquitectura a toda la ciudadanía como parte de la formación cívica de las personas, nos llevó a estudiar el fenómeno en Finlandia y en los otros países que poseen experiencias similares, para luego tener una reflexión asociada a nuestro contexto.

En Finlandia este fenómeno había empezado a surgir en muchos lugares a la vez, sin políticas concretas al respecto, como una tendencia general para enfrentar las cuestiones de las personas y su vínculo con su medio. La Asociación de Arquitectos de Finlandia (SAFA), reunió en el año 2001 todas estas experiencias en un documento titulado "Discovering Architecture"1, comenzando con las siguientes palabras del Ministro de Cultura de aquel tiempo, Suvi Linden:

\begin{abstract}
Todos los ciudadanos debieran tener un entendimiento activo del medio ambiente construido, independiente de la edad, ocupación o formación educacional. Cada uno de nosotros va a tener que tomar una posición en algún minuto acerca de temas relacionados al medio ambiente construido, y por ende, tener opinión sobre la calidad de nuestras propias vidas y la de los demás.
\end{abstract}

La apreciación de la arquitectura en Finlandia es de muchas maneras ejemplar. En los años 1980, cuando la educación general en arquitectura se tornó un tema de seria discusión, Finlandia, junto con otros países nórdicos, lanzaron su primer proyecto experimental sobre el tema. Desde entonces, proveer educación cívica en arquitectura se ha ganado fama internacional también, a pesar de que tal educación sea implementada principalmente a niños. A fin de producir realmente alta calidad, la industria de la construcción debe tener clientes educados y entusiastas que saben de lo que están hablando y que lo que ellos pueden y deben demandar acerca de los edificios que ellos van a utilizar.

La Ordenanza de Urbanismo y construcciones que se acaba de aprobar, requiere que las personas avancen a un nivel de maestría y entendimiento del medio ambiente construido, y les crea una oportunidad y una obligación. La responsabilidad de defender el interés de los ciudadanos recaía solamente en expertos y la sociedad civil, pero ahora se ha extendido a todos los fineses, quienes necesitan adquirir las habilidades y maestrías necesarias para tomar esta responsabilidad.

A partir de nuestra experiencia decidimos realizar el seminario de investigación tutelada correspondiente al quinto año de la Carrera de Arquitectura en la F.A.U. de la Universidad de Chile ${ }^{2}$, sobre el tema: Educación Cívica en Arquitectura, directrices para la formación de ciudadanos con conciencia en arquitectura. Este seminario recoge gran parte de nuestro conocimiento adquirido en Finlandia. También nuestras vivencias como "docentes en arquitectura", de forma experimental, de 24 niños de $4^{\circ}$ Básico del colegio Miguel Cruchaga Tocornal de la Sociedad Protectora de la Infancia (ciudad de Puente Alto, Región Metropolitana de Santiago de Chile) dentro de la asignatura de "Tecnología".

En Finlandia contactamos a las tres escuelas principales que están desarrollando este tipo de educación: en Helsinki existe Arkki, donde tuvimos como interlocutora a su creadora Pihla Meskanen; en el norte, en Lapinlahti, está la escuela llamada LASTU, en la que dialogamos con su creador Annu Puurunen y con la profesora jefa Mervi Eskelinen, finalmente, estuvimos en la Escuela de Artes Visuales de Jyväskyla - ciudad del Arqto. Alvar Aalto- donde nos recibió Ilpo Vuorela.

La experiencia de Puente Alto nos enfrentó a la implementación de lo estudiado anteriormente en el país del norte -elaborando una propuesta propia de ejercicios y proyectos- pero, por sobre todo, al hecho de tener que asumir la docencia no

\footnotetext{
${ }^{1}$ http://www.safa.fi/archive/311_discovering-achitecture.pdf

2 Prof. Guía Arqto. Ernesto Calderón A.
} 
siendo pedagogos. De gran ayuda fue la labor brindada por el Inspector General del colegio J orge Abarca, y por profesores que nos apoyaron tanto en visitas guiadas, como en las labores dentro del colegio. Gran parte de nuestra propuesta se basó en nuestra formación universitaria, adaptándola, resaltando aspectos que considerábamos importantes, como por ejemplo el trabajo en grupo, la comprensión espacial y del medio, la valoración de nuestro patrimonio y el proceso creativo, entre otros.

Este tipo de experiencias nos permitió comprender las similitudes y diferencias del trabajo que realizan los niños de culturas distintas, así como comprender las distintas etapas cognitivas en el proceso de aprendizaje. También tuvimos que enfrentar la poca costumbre de los niños a métodos de aprendizaje más "libres", pero por lo mismo, más responsables. El hecho de realizar trabajos con nuevas técnicas, así como con nuevas materialidades, conocidas pero no utilizadas, abrió nuevas formas de expresión a los niños para sus trabajos, además de ayudar al desarrollo de las actividades de los otros cursos. Todo esto lo pudimos captar al finalizar el proceso, en los resultados de las evaluaciones finales realizadas a otros profesores y a los apoderados.

\section{1.- Objetivos e hipótesis}

El objetivo principal de nuestro trabajo fue contribuir a dar cuenta de la necesidad en Chile de educar a los ciudadanos en arquitectura, en la etapa escolar, para que puedan tener herramientas y un lenguaje adecuado que les permita ser partícipes de las instancias de diálogo y toma de decisiones para la conformación de su propio Medio Ambiente Construido, además de ser ciudadanos críticos frente a la arquitectura, en el marco de la construcción de nuestra democracia.

Basándonos en los casos estudiados en Finlandia, establecimos una hipótesis general sobre el tema, orientado a la aplicación en nuestro país.

\section{HI PÓTESI S GENERAL}

Educar en arquitectura y urbanismo en la niñez, durante el proceso de comprensión del Medio Ambiente Construido (MAC), permitiría constituir un proceso educativo para contribuir a conformar ciudadanos más conocedores e interesados por los temas del MAC, que manejen una serie de conceptos y habilidades que les permitan ser partícipes de su construcción, esto es: participar en la confección de los planes reguladores, ser clientes más informados frente a la oferta inmobiliaria, ser ciudadanos conscientes y educados en su actuar en el espacio público y valorar el patrimonio. La existencia de ciudadanos con conciencia en arquitectura y urbanismo, fortalecería los lazos que relacionan a los actores participantes de la creación del MAC - arquitectos, ciudadanos y autoridades - permitiendo el desarrollo de un lenguaje común y mejorando el diálogo en las instancias de toma de decisiones que le dan forma. A pesar de que existen algunos ejemplos en el mundo de programas y experiencias de Educación Cívica en Arquitectura, éste debe estar moldeado por la cultura y motivaciones de la propia sociedad en la que se desarrolla, en este caso Chile.

Esta hipótesis no era comprobable en el plazo de un semestre académico, ya que consiste en una reforma educativa, lo que arroja resultados después de años de implementación. Por ello, y a raíz de la oportunidad que se nos presentó de llevar a cabo el proyecto en la Sociedad Protectora de la Infancia, establecimos preguntas específicas, que dieron pie a dos hipótesis específicas, posibles de abarcar en el tiempo de un seminario de investigación en la academia y en una experiencia empírica asociada. 
La propuesta de la hipótesis se centra en definir el contexto teórico dentro del que se posiciona el planteamiento de la educación cívica en arquitectura, como son las dinámicas sociales que se dan en las tomas de decisiones que afectan al medio ambiente construido (Fig.1):

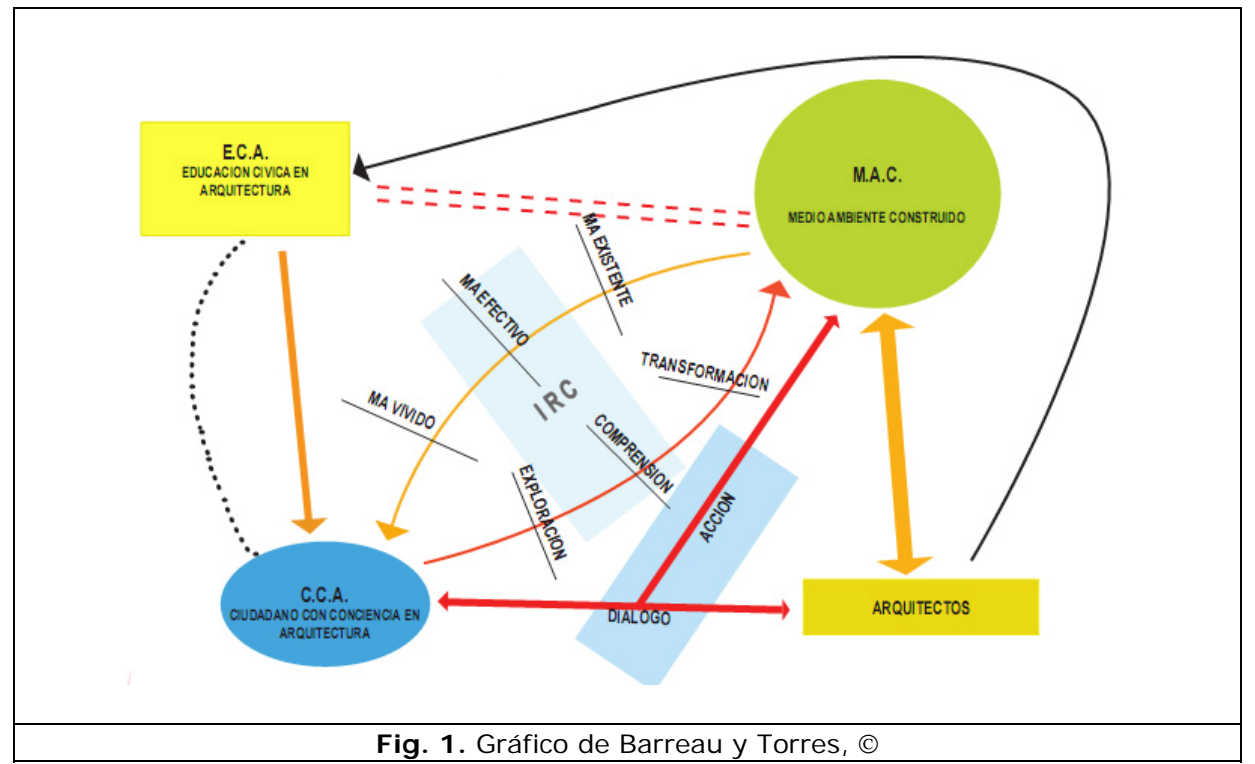

Acerca de ello surge el primer objetivo específico:

a. Dar cuenta del Sistema de Relaciones Ciudadano-Arquitectónicas (SRCA) que se nutre de la Educación Cívica en Arquitectura, que involucra al vínculo de dialogo y acción del ciudadano con el arquitecto en la toma de decisiones que conciernen al Medio Ambiente Construido (MAC) e incorpora el vínculo de interacción con retroalimentación compleja (IRC) que establece con su Medio Ambiente Construido.

Establecimos los principales actores participantes en las dinámicas que dan forma al medio y las relaciones que establecen (Fig.2):

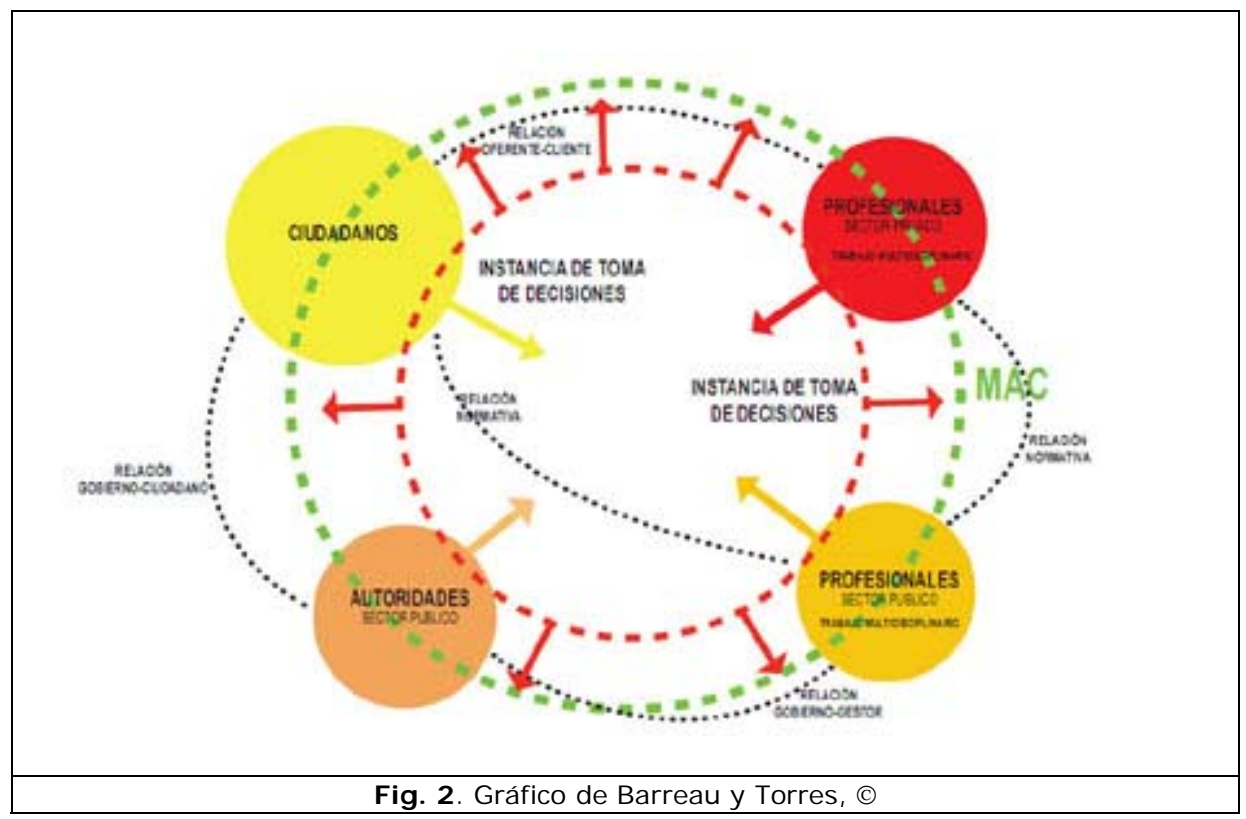

Fig. 2. Gráfico de Barreau y Torres, (c) 
El segundo tema fundamental de la hipótesis general se concentra en la educación en la niñez, puesto que en dicha etapa las personas desarrollamos el principal vínculo con el medio. Este es el período más rico e intenso de exploración y descubrimiento de lo que nos rodea, por lo que tiene un potencial enorme en términos de sensibilización respecto del Medio Ambiente Construido. De acuerdo a palabras de Howard Andrews, en su libro Ecología social de la niñez urbana: hacia una perspectiva comparativa:

Aun cuando podríamos decir que nuestro interés por viajar y explorar nunca realmente se detiene durante la extensión de nuestras vidas, la peculiar mezcla de curiosidad, imaginación y despertar de la independencia del individuo, desde la temprana niñez hasta la adolescencia, hacen que este período de nuestras vidas se caracterice por una intensa exploración.

Los siguientes objetivos específicos hacen referencia de ello:

b. Dar cuenta del tipo de relación que establece el habitante con su Medio Ambiente Construido (IRC), comprendiendo los tipos de actividad mental en el proceso de comprensión del MAC y sus dimensiones cognitivas, a fin de poder sentar una base sobre la cual definir una estrategia de contenidos y métodos pedagógico adecuado para la enseñanza cívica de la arquitectura en niños en etapa escolar.

c. Dar cuenta de los procesos de apropiación particulares de los niños al entablar su relación de exploración, comprensión y transformación con el Medio Ambiente Construido, los medios que utiliza naturalmente para explorarlo y los medios que posee la educación cívica en arquitectura y urbanismo para agudizar dicho proceso. Esto constituiría un acercamiento a la puesta en práctica del programa la educación cívica en arquitectura en Puente Alto.

d. Proponer las directrices relacionales para la formación de un Programa de Educación Cívica en Arquitectura para niños chilenos, que sea comprensible para los arquitectos y profesionales responsables de la conformación del Medio Ambiente Construido, a fin de que puedan involucrarse en los procesos de educación de los ciudadanos, a la vez que sea entendible para las autoridades y profesionales del área de la educación, a fin de poder guiar su implementación pedagógica.

Las preguntas específicas que se desprenden de la hipótesis se orientan entonces hacia la niñez, a pesar de que el tema del seminario, en otra instancia, pudo albergar la relación de los adultos con su medio, pero debido a la oportunidad de llevarlo a cabo, desarrollamos las hipótesis específicas que enmarcan el campo de estudio al área de la niñez.

\section{PREGUNTA 1}

¿De qué manera se aproxima un niño en etapa escolar, en su experiencia de exploración, comprensión y transformación del medio que lo rodea, a entablar un diálogo físico, perceptual y cognitivo con su Medio Ambiente Construido?

\section{HI PÓTESI S ESPECÍ FI CA 1}

El niño entabla su relación física, perceptual y cognitiva con el Medio Ambiente Construido en un proceso de exploración a través de todos sus sentidos y sensaciones, a través del movimiento, la manipulación de objetos, la creación de sus propios espacios y principalmente mediante el juego. Esto ocurre desde el comienzo de la movilidad personal de gatear en el suelo, hasta la libertad de la experiencia de caminar por la ciudad en la adolescencia. El niño, de acuerdo a la edad, entabla una relación simbólica con el Medio Ambiente 
Construido, siendo para ello esencial la socialización del niño en los espacios públicos y privados, el paso del tiempo en su experiencia de crecimiento y en la construcción de estas estructuras mentales significativas (o estructuras cognitivas) y la construcción de mundos propios. Mediante esto el niño se va apropiando de su entorno y desarrollando una identidad con su medio, parte esencial de la formación de ciudadanos concientes en arquitectura en la adultez.

\section{PREGUNTA 2}

¿Mediante qué medios y metodologías de la enseñanza de la arquitectura podría la educación cívica en arquitectura enriquecer y potenciar este proceso de exploración física, perceptual y cognitiva del niño con su Medio Ambiente Construido?

\section{HI PÓTESI S ESPECÍ FI CA 2}

La educación, comprendida como la evolución de las estructuras perceptuales y cognitivas ya existentes, es la forma de incentivar a que este proceso de descubrimiento del niño se amplíe progresivamente, abarcando distintas dimensiones del Medio Ambiente Construido ${ }^{3}$, desde el Medio Ambiente Existente (MAEx), hasta el Efectivo (MAEf) y luego hacia la complejidad del Medio Ambiente Vivido (MAV). De acuerdo al entendimiento de los medios arquitectónicos que posibilitan la exploración del espacio, como la maqueta, el dibujo y el uso de programas digitales, se agudiza la capacidad de observación y comprensión del MAC, lo que sienta las bases para la asimilación de habilidades ciudadanas más específicas en la adultez.

\section{2.- Aspectos pedagógicos}

Sobre los aspectos netamente ligados a la pedagogía, nos apoyamos en los principios del psicólogo suizo Jean Piaget, de quien rescatamos la teoría básica que correspondía al desarrollo cognitivo de un niño de entre 8 y 10 años, que corresponde a la etapa que define como de "Operaciones Concretas". Se establece que en esta etapa es muy importante la experimentación con objetos, ya que los niños no obedecen tanto a enunciados verbales u hipótesis, sino que directamente a los elementos. Se encuentran en un proceso de descubrimiento muy complejo y ligado a lo concreto, aspecto que también destacó el profesor Jorge Abarca, Inspector General del colegio, al dar inicio a las clases con los niños.

La acogida de los niños fue muy buena, pudimos apreciar el entusiasmo que generó tanto en ellos como en sus profesores y apoderados. A pesar de no tener formación en pedagogía, era evidente la conexión que se establecía entre los niños y los ejercicios que fuimos realizando. Ellos lograban concentrarse en lo que se les pedía e incluso comenzaron a agregar elementos e historias que no estaban indicadas.

De acuerdo a lo rescatado en las conversaciones que establecimos con Pihla Meskanen (Arkki), Mervi Eskelinen (LASTU) y Ilpo Vuorela (Escuela de Artes Visuales de Yyväskyla) la enseñanza de la arquitectura tiene la virtud de poner atención en el proceso más que en el resultado, lo que permite trabajar con los niños sobre sus propias habilidades y desarrollo cognitivo por sobre las características estéticas de los trabajos. A raíz de ello se establece una diferencia crucial entre el trabajo de un profesor de arte y el de un arquitecto, ya que lo que se busca con la educación cívica en arquitectura es la formación de ciudadanos con conciencia en arquitectura, y no arquitectos o artistas.

Los trabajos que realizamos con los niños se basaron principalmente en el proceso educativo, comenzando a explorar materialidades y formas asociadas a historias y personajes que iban creando los niños. Lo abordamos desde las distintas escalas en

\footnotetext{
${ }^{3}$ TRIEB, Michael. Seminario Diseño Urbano, Teoría y Práctica, Santiago: Universidad de Chile, Facultad de Arquitectura
} y Urbanismo, 1983, 58 h, DOCUMENTOS AQ06, Manuel Fernández. 
maquetas de manera progresiva, es decir, desde modelos a escala 1:100 aproximadamente, hasta modelos a escala $1: 1$, en que los niños pudieron experimentar el lugar desde su propia experiencia. Después de cada trabajo, había un ejercicio de comentarios, para intentar vislumbrar qué es lo que habían aprendido los niños, y, a la vez, realizar observaciones complementarias focalizadas en nuevos aspectos, para incrementar la capacidad de observación Figs.3-4).

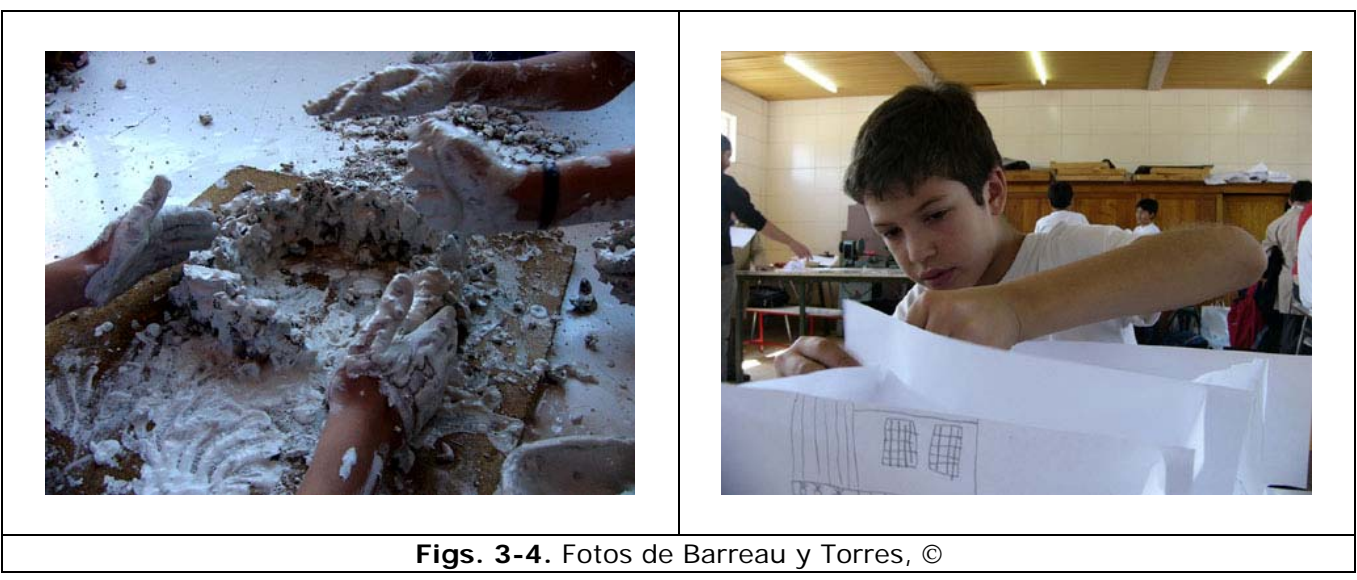

El trabajo en equipo fue otro de los pilares fundamentales de los ejercicios, puesto que en el sistema de educación tradicional no tiene mucha cabida, pero en arquitectura es fundamental ya que potencia el diálogo entre pares, desarrolla las capacidades para ponerse de acuerdo, para respetar las opiniones de los compañeros, para organizarse, asumir roles y, en suma, trabajar en conjunto por un objetivo común. Enfocamos el proceso de creación y aprendizaje como un desarrollo colectivo, en que se complementan las virtudes de cada uno de los niños y se aprende de los compañeros.

Un ejemplo fue un ejercicio en que construimos espacios escala 1:1. Había que formar equipos, ponerse un nombre y desarrollar el lugar de reuniones para ellos; por ende, había que elegir un lugar y definir cómo iba a ser para que les permitiera conversar tranquilamente (Fig.5).

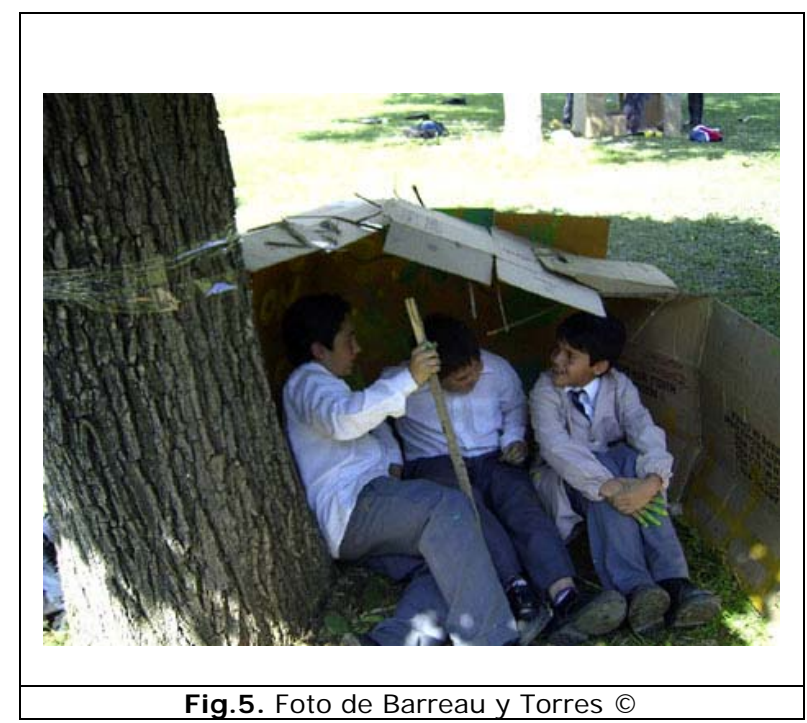

Fig.5. Foto de Barreau y Torres $\odot$ 
Lo observado sobre el comportamiento natural que presenta un niño al jugar en su entorno por los arquitectos en Finlandia, es una tendencia a generar espacios, lugares donde realizar sus juegos. Intentamos también potenciar esta condición trabajando en lugares del colegio elegidos por los niños, a fin de que les naciera crear nuevos espacios en ellos. Notamos que los lugares preferidos eran los que tenían acceso restringido para los alumnos, a la vez que aquellos que estaban ocultos de los ojos de sus profesores, con calidad de refugios. En cada lugar los niños tenían distintas historias, lo que tratamos de resaltar al conversar y trabajar con ellos.

\section{3.- Experiencia}

Realizamos las clases en la Escuela Miguel Cruchaga Tocornal de Puente Alto en el horario asignado para la asignatura de "Tecnología", que era dos días a la semana en las tardes. Dictamos las clases durante tres meses, lo que nos permitió conocer bastante a los niños y llevar a cabo varios ejercicios diseñados con objetivos bien específicos.

Podríamos clasificar cuatro distintos tipos de actividades para estimular el proceso de aprendizaje en los niños:

a) El trabajo con maquetas en las distintas escalas mencionadas, lo que permitió un descubrimiento directo de las formas de construir con los distintos materiales. Esta experiencia fue muy buena para que los alumnos se concentraran en una tarea, comprendiendo su funcionamiento mediante la interacción con el objeto.

b) La muestra de una película llamada "Baraka", en que se muestran distintas culturas y su arquitectura característica. Lo principal de la película es que poseía muchas imágenes de realidades distintas y sonidos que queríamos que estimularan los sentidos y la sensaciones de los niños.

c) Visita a la Bienal de Arquitectura de Santiago 2006, paseo que también incluyó el Palacio de la Moneda, la Plaza de la Constitución y la Casa Central de la Universidad de Chile. En esta experiencia quisimos que los niños conocieran la exposición de la Bienal y pudieran ver las proyectos que estaban haciendo los arquitectos chilenos, además de que conocieran el edificio y los alrededores de la Moneda, en una vivencia directa con la ciudad (Fig.6).

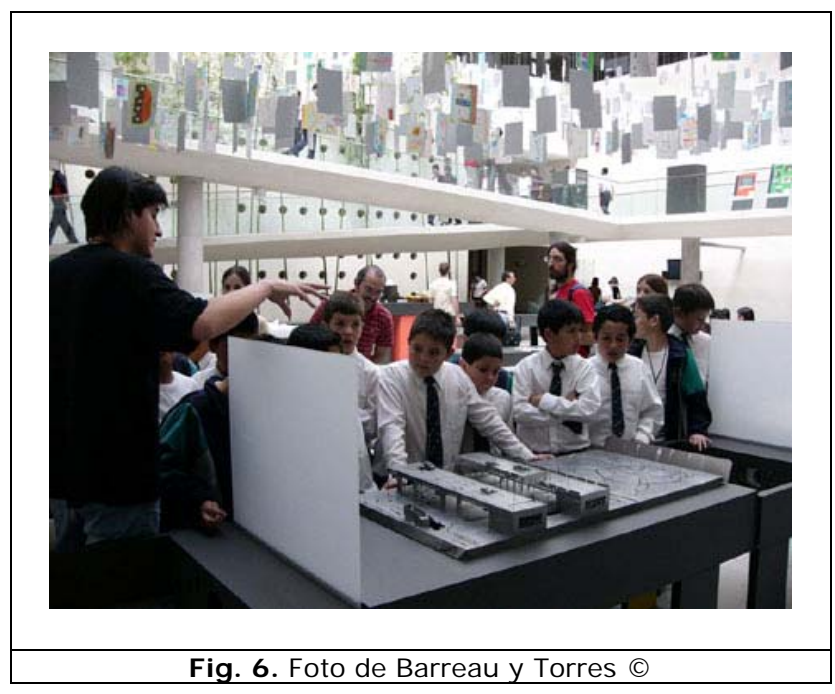


d) Un trabajo en computadores con el programa de modelación 3D Sketchup, con el fin de que los niños tuvieran la experiencia de usar el computador que usualmente usan para navegar en Internet, como una herramienta para efectos tridimensionales (Fig.7).

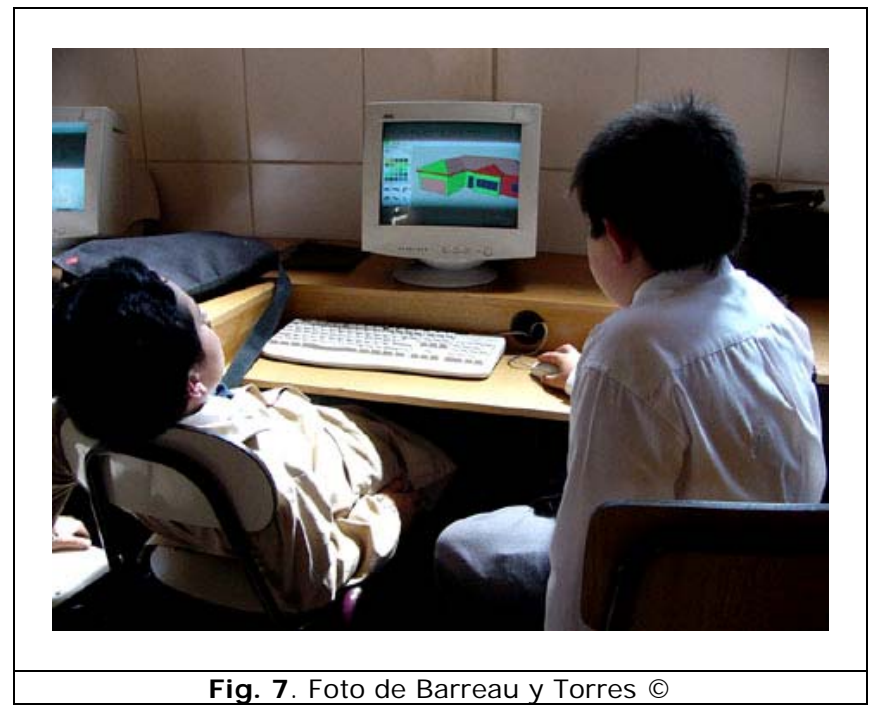

\section{4.- Resultados}

La experiencia de hacer clases en un colegio e implementar una metodología basada en una propuesta teórica desde nuestra perspectiva de arquitectos, constituyó una vivencia enriquecedora para nuestra formación de ciudadanos arquitectos, a la vez que un gran desafío al asumir el rol de docentes, sin poseer formación en pedagogía.

Desde el planteamiento de la "formación de ciudadanos en arquitectura" hasta la realidad de la sala de clases existe un gran salto, ya que el manejo con los niños es bastante complejo y realizar la evaluación pertinente para conocer nuestro desempeño y el aprendizaje de los niños nos fue muy difícil, ya que desconocíamos mecanismos adecuados para ello.

Este problema nos llevó a realizar las clases a partir de los conceptos que habíamos abordado en el marco teórico del seminario y sobre la experiencia que conocimos en Finlandia, y no desde el reconocimiento del nivel de aprendizaje de los niños. Nos era difícil detectar los conceptos que ellos iban asumiendo, incluso cuando discutíamos los proyectos, al final de cada uno de ellos, debido al nivel de expresión verbal de los niños de esa edad, no lográbamos hacer un diagnóstico claro sobre los avances.

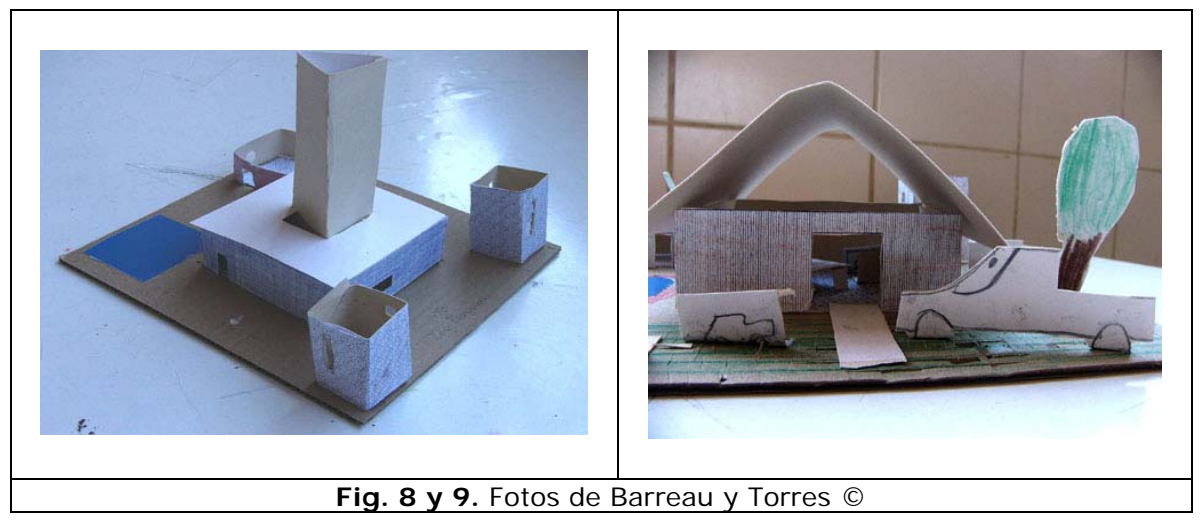


Nos dimos cuenta que el lenguaje que utilizamos los arquitectos es muy difícil de entender, a pesar de referirse a conceptos y elementos que pueden ser descritos por cualquier persona. Si es que este rol educador del arquitecto se quisiera potenciar, se debiera estudiar el lenguaje con el que pretendamos establecer un diálogo con la ciudadanía, ya que de lo contrario, podríamos no comunicar lo que quisiéramos.

La acogida que tuvo la experiencia, tanto entre profesores, apoderados y alumnos, fue muy buena. Mediante una pequeña encuesta se hizo notar la conformidad y entusiasmo que se había generado a lo largo de la experiencia. Uno de los resultados que nos sorprendió fue que los niños habían subido sus notas en la asignatura de educación matemática. Esto abre otro campo de investigación en torno a las habilidades que desarrolla este tipo de educación, basado en los conceptos básicos con los que se enseña arquitectura, lo que podría tener una potencialidad insospechada.
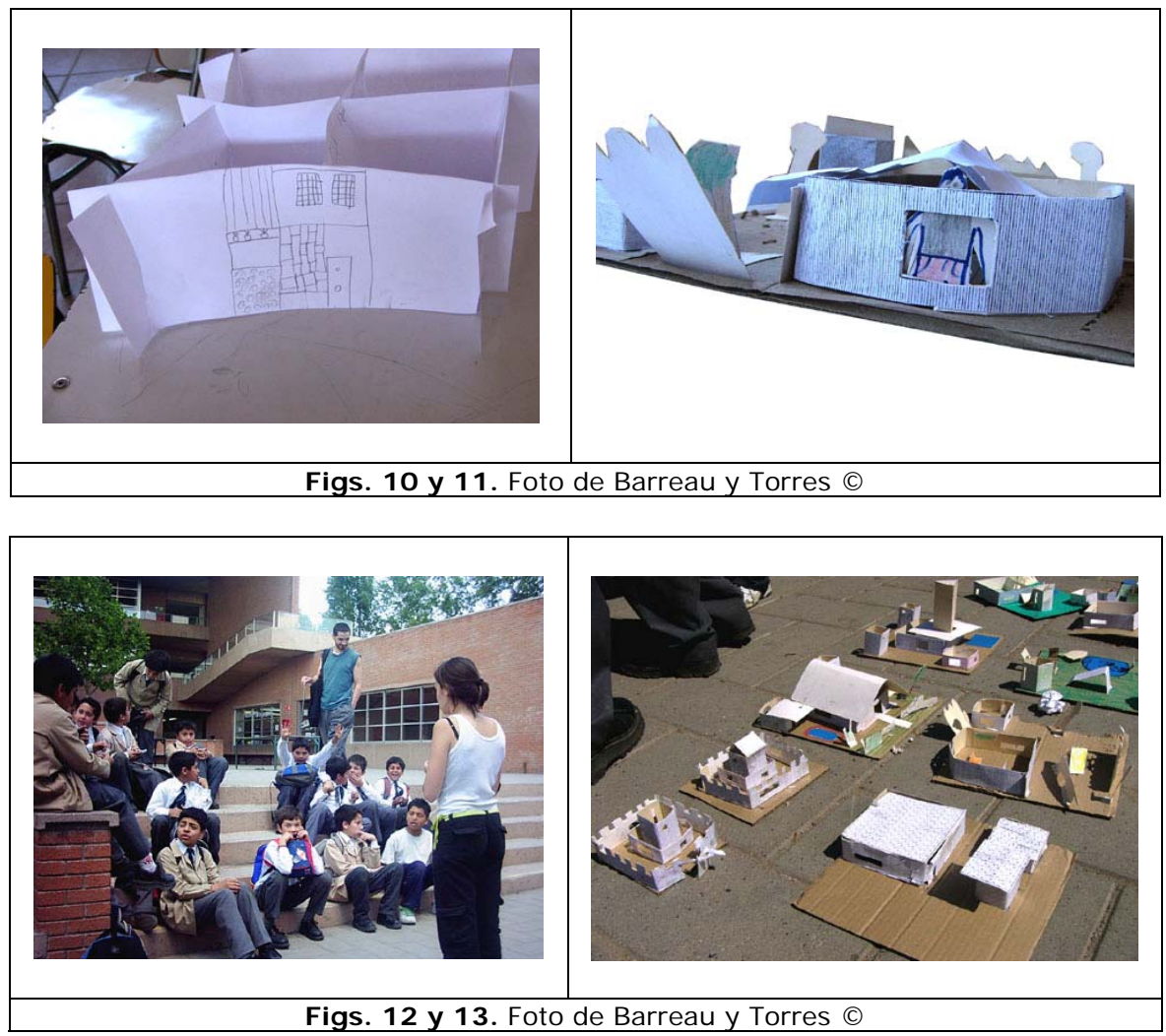

\section{5.- Reflexiones finales}

Como estudiantes de la Universidad de Chile, creemos que la experiencia y la investigación sobre temas de educación ligados a nuestra profesión, fue un gran proceso de aprendizaje también para nosotros, puesto que nos hizo ver la arquitectura desde el punto de vista del habitante y más allá de eso, desde la mirada de los niños. Nos hemos acercado a entender la importancia de la arquitectura como fenómeno, más que como disciplina, reflexionando sobre nuestro rol en la sociedad y específicamente sobre el rol educador que nos corresponde a todos como miembros de ella.

La arquitectura en sí educa y hemos podido acercarnos a diversas teorías sobre la ciudad educadora, especialmente mediante Josef Muntañola. Existe un gran movimiento mundial para potenciar el rol educador de las ciudades, ligadas a proyectos de municipios o de organizaciones civiles. Todas ellas reconocen la 
necesidad de educar la experiencia urbana, y que el lenguaje de las ciudades no dice nada a nadie sin un conocimiento previo. Por ello se hace indispensable la educación de las personas sobre su propio medio.

En este sentido, consideramos igualmente importante un comportamiento cívico activo - por ejemplo la participación en las instancias de toma de decisiones concernientes a la ciudad- como el comportamiento pasivo, pero colaborante con una buena convivencia y cuidado de su medio ambiente natural y construido.

Indiscutiblemente la educación juega un rol esencial en el desarrollo del país y en la convivencia de sus ciudadanos, esto ha quedado más claro aun después del discurso presidencial de este 21 de mayo (M. Bachelet, 2007), en que se ha destinado el 0.5 del superávit fiscal a mejorar la educación básica y media, pilar fundamental para el desarrollo del capital humano.

Grandes transformaciones como el proceso de descontaminación de las ciudades requieren de hábitos, de personas concientes del funcionamiento urbano y de las consecuencias que cada uno de sus actos trae a la ciudad. La concepción de que para poder descongestionar la ciudad de Santiago es necesario que más personas dejen sus automóviles particulares y se incorporen al transporte público, es otro tema que requiere de un entendimiento más profundo de nuestra relación con la ciudad, lo que podría tener mejor aceptación si los ciudadanos tuvieran una mejor formación cívica en arquitectura y urbanismo.

Esa mirada conocedora de la arquitectura y el urbanismo podría ser lo necesario para permitir la innovación y el desarrollo de la arquitectura y el urbanismo nacional, si lográramos sintonizar al habitante con los creadores de los edificios y espacios públicos, así como promover la opinión y participación en el proceso de crear ciudad.

\section{6.- Bibliografía}

Libros:

-ANDREWS, Howard, Ecología Social de la niñez urbana: hacia una perspectiva comparativa, 1987.

-BERGER, Peter y LUCKMAN, Thomas, La construcción social de la realidad, Buenos Aires: Amorrortu editores, 1999, 233 págs. ISBN 9505180098.

-NOVO, María, Análisis Interdisciplinar de la problemática Ambiental (dirigido por María Novo; coordinador Ramón Lara), Madrid, Fundación Universidad-Empresa, 1997. ISBN 8478421467 (obra completa).

-RAPOPORT, Amos, Aspectos Humanos de la Forma Urbana: hacia una confrontación de las ciencias sociales con el diseño de la forma urbana, Barcelona (versión castellana de Joseph Muntañola), Barcelona, Editorial Gustavo Gili, 1978, 381 págs. ISBN 8425207185.

-RAPOPORT, Amos, Aspectos de la Calidad del Entorno (traducción Justo G. Beramendi), Barcelona, Editorial La Gaya Ciencia S.A., 1974, 127 p., ISBN 8470804146.

Seminarios de investigación tutelada, $\mathrm{V}$ año carrera de Arquitecto, F.A.U. U. de Chile: 
-BARREAU DALY, Camila; TORRES GÁLVEZ, Juan Luis, Educación cívica en arquitectura: directrices para la formación de ciudadanos con conciencia en arquitectura. Seminario de Investigación tutelada, V año carrera de Arquitectura, Facultad de Arquitectura y Urbanismo, Universidad de Chile, Prof. Guía. Arqto. Ernesto Calderón Álvarez, 2006. [E-mail:camila.barreau@gmail.com]

-BRAVO COX, Fernando. Niños / Ciudad / Arquitectura: hacia un aprendizaje y sensibilización de la ciudad. Profesor Guía: Dra. Arqta. María Bertrand S. TESIS $\mathrm{UCH}, 110$ p. Facultad de Arquitectura y Urbanismo, Universidad de Chile, Santiago, 2004.

-RIQUELME, Omar, Espacio Público en la educación cívica: educación escolar frente al tema del espacio público. Para una actitud cívica consciente en la instancia ocupacional y planificadora del espacio público: resultados de una experiencia con estudiantes de 1o. de enseñanza media. Profesor Guía: Dra. Arqta. María Bertrand S. TESIS UCH, 200 p. Facultad de Arquitectura y Urbanismo, Universidad de Chile, Santiago, 2000.

Revistas:

CA, $N^{\circ} 127$ - CIUDADANíA. Revista editada por el Colegio de Arquitectos de Chile A.G., 2006.

\section{Documentos en línea:}

-BITAR, Sergio, Ministro de Educación de Chile. Discurso en Inauguración Seminario "Fortalecimiento de la democracia a través de la educación ciudadana". Editado por: Centro de Extensión de la P. Universidad Católica de Chile, 26 de Agosto, 2004.

www.mineduc.cl/biblio/documento/SEMINARIO_EDUCACION_CIVICA_(agosto).doc

-BORJA SEBASTIÀ, Jordi. "La ciudad conquistada. Un punto de vista de la sociología". En La Ciudad Educadora = La Ville Éducatrice. Ajuntament de Barcelona. I Congrés Internacional de Ciutats Educadores. Barcelona : Ajuntament de Barcelona , 1990. pp. 49-28. [Contribución en monografía] ISBN 84-7609-398-5.

-KORPELAINEN, Heini y YANAR, Anu. Discovering Architecture. The Finnish Association of Architects and the Finish Arts Council of Finland. Art-Print Oy, Helsinki, 2001. ISBN 951-9357-10-9

-MATA GARRIGA, Marta. "La institución escolar y la ciudad". En La Ciudad Educadora $=$ La Ville Éducatrice. Ajuntament de Barcelona. I Congrés Internacional de Ciutats Educadores. Barcelona: Ajuntament de Barcelona, 1990. pp. 233-244. [Contribución en monografía] ISBN 84-7609-398-5.

-MUNTAÑOLA, Joseph. "La ciudad educadora desde la arquitectura". En La Ciudad Educadora = La Ville Éducatrice. Ajuntament de Barcelona. I Congrés Internacional de Ciutats Educadores. Barcelona: Ajuntament de Barcelona, 1990. pp. 83-89. [Contribución en monografía], ISBN 84-7609-398-5.

-PUIG ROVIRA, Joseph. "La ciudad y el desarrollo moral y cívico". En La Ciudad Educadora $=$ La Ville Éducatrice. Ajuntament de Barcelona. I Congrés Internacional de Ciutats Educadores. Barcelona: Ajuntament de Barcelona, 1990. pp. 185-196. [Contribución en monografía] ISBN 84-7609-398-5.

-TRIEB, Michael. Seminario Diseño urbano, teoría y Práctica. Santiago: Universidad de Chile, Facultad de Arquitectura y Urbanismo, 1983, 58 h, DOCUMENTOS AQ06, Manuel Fernández. 


\section{Sitios en Internet:}

- Arkki, Architecture School for Children and Youth: www.arkki.net

- Lastu School (en finlandés): www.saunalahti.fi/lastu2

- Visual Arts School in Jyväskylä: www.jkl.fi/kulttuuri/kuvataidekoulu

- Museo Alvar Aalto: www.alvaraalto.fi/museum

- MFA, Museo de Arquitectura de Finlandia: www.mfa.fi

- Playce, Asociación Internacional de Educación en Arquitectura: http://www. playce.org/

- Sitio Web de la Arquitectura Finlandeza: www. finnisharchitecture.fi

- Fundación Alvar Aalto: www.alvaraalto.fi/foundation

- Arkitekturmuseet, Suecia: www.arkitekturmuseet.se

- English Heritage Education: www. HeritageEducation.net

- Lighthouse, Escocia: www.rias.org.uk

- Cebe, Centro para la Educación sobre el Medio Ambiente Construido: http://cebe.cf.ac.uk

- http://www.wikipedia.org

- MINEDUC, Chile: www.mineduc.cl

Para comunicación con los autores:

$<$ camila.barreau@gmail.com>

<ltorres@live.com> 